\title{
Design of model reference adaptive control for fast steering mirror based on generalized error fast differential method
}

\author{
Ai Zhiwei ${ }^{a}$, Ji Jianbob ${ }^{b}$, Wang Pengjuc, Liang Ruijing ${ }^{d}$, Zhu Hua \\ Guilin University of Aerospace Technology, Guilin 541004, China \\ aaizhiwei752@163.com, bjijianbo@guat.edu.cn, 'wangpengju@163.com, ${ }^{\mathrm{d}} 936931715 @ q q . c o m,{ }^{\text {e}} 838148216$ \\ @qq.com
}

Keywords: FSM; flexible link; adaptive control; fast differential; incomplete derivative PID

\begin{abstract}
Due to the existence of flexible link in fast steering mirror (FSM), the uncertainty of the structural stiffness of the flexible-supported FSM is greatly increased when FSM works. This leads to a higher requirement for the robustness of control system. Aimed at this problem, a generalized error fast differential based adaptive controller is proposed by combining the adaptive control theory with the tracking differential theory. The controller can make appropriate adjustments to track the given reference model if the structure changes. Firstly, the mathematical model of the FSM control system is optimized to match the mathematical model of the tracking differentiator. Secondly, according to the input and output data of the FSM, the adaptive control system is designed based on Lyapunov stabilized theory. Finally, the denoised system's generalized error signal and its differential signal are obtained by using a fast differentiator. The signals are used in adaptive control system immediately. The numerical simulations results show that the proposed algorithm can both suppress the influence of the change of structural stiffness and the sensor's noise.
\end{abstract}

\section{Introduction}

To insure the stabilization of the visual axis of the optics-electronics equipment, and achieving stable tracking and imaging, fast steering mirror (FSM) is widely used between the motion carrier and the optics-electronics equipment to isolate the impact of the motion carrier on the photoelectric equipment to acquire high-precision tracking data ${ }^{[1-2]}$.

According to the difference of support structures, the styles of FSM mainly have two kinds: frame and flexible. The FSM with flexible support is widely used in the optics-electronics systems which require high precision and small stroke and use voice coil motors (VCM) or piezoelectric ceramics as its driver. In recent years, with the development of aerospace technology, high-precision FSM control methods have been greatly developed, and many advanced control strategies have emerged to improve the control performance.

A two-step control strategy is proposed for the unmolded part of the gyro-stabilized platform model, the random disturbance and the measurement noise of the output signal in [3]. In the first step, the unmolded part of the system is observed and feed-forward compensation by using active disturbance rejection control. In the second step, Kalman filter is used to filter the state disturbance and measurement noise in the system. An acceleration feedback technique is proposed in [4] to improve the initiative suppression ability of inertial stabilized platform. An active disturbance rejection control strategy based on electric current loop is proposed in [5] to improve the disturbance rejection ability of aviation optics-electronic stabilized Platform. A three loop control system is proposed in [6] to suppress the influence of the unbalanced disturbance on inertial stabilized platform. All of these above referred control strategies contribute to the precision of the control system, as well as lowering its response speed. The increased use of sensors is not only cost more but also introduce more measurement noise.

Because of the existence of flexible link, the uncertainty of the structural stiffness of the flexible-supported FSM is greatly increased when platform works. It is necessary to adjust the 
controller after the change. The incomplete derivative PID control parameters can be obtained based on improving root-locus algorithm for VCM driven fast steering mirror with flexible structure in Ref. [7] and the results show the superiority. The reference model of the inertial stabilized platform can also be get by this algorithm. On the basis of the ideal reference model, the decision towards the expected system performance will be made according to comparison result between the actual model and the ideal reference model. Performance of the actual system will tend to the expected value by adjusting the control parameter properly and then realizing the adaptive control. The generalized error signal with noise will be treated by using fast differentiator. The treated generalized error signal and its derivation signal will be introduced to the adaptive control system to realize the adaptive control algorithm based on generalized error fast differential.

\section{Model treatment}

The researched FSM is composed of base, flexible link, VCM and load. There are two kinematic axis of the platform. It is feasible to research one kinematic axis because of the good symmetry of the structure. The math model of the researched FSM can be decomposed into a inertial link and a oscillating link, expressed as which is expressed as:

$$
G(s)=\frac{k_{g}}{\left(p_{2} s^{2}+p_{3} s+1\right)\left(p_{4} s+1\right)}
$$

Here, $k_{g}, p_{2}, p_{3}, p_{4}$ are defined by the FSM itself. To eliminate the influence of the oscillating link, the incomplete derivative PID control is introduced into the control system. The transfer function of incomplete derivative PID control is given as follow:

$$
C(s)=k_{p}+k_{i} \frac{1}{s}+\frac{k_{d} s}{T_{f} s+1}
$$

Here, $k_{p}, k_{i}, k_{d}, T_{f}$ are the given coefficients of proportion link, integral link, differential link and the constant value of inertial link. To insure the introduction of the controller can eliminate the influence of oscillating link, the incomplete derivative PID controller need to satisfy the following relationship:

$$
C(s)=\frac{k_{c}\left(p_{2} s^{2}+p_{3} s+1\right)}{s\left(T_{f} s+1\right)}
$$

The open loop transfer function can be expressed as follow:

$$
L(s)=C(s) G(s)=\frac{k_{c} k_{g}}{s\left(T_{f} s+1\right)\left(p_{4} s+1\right)}
$$

Because of $T_{f} p_{4} \ll\left(T_{f}+p_{4}\right)$, the order of the actual model can be lowered by neglecting the coefficient of highest order. The transfer function turns to following:

$$
L(s)=\frac{k_{c} k_{g}}{\left(T_{f}+p_{4}\right) s^{2}+s}
$$



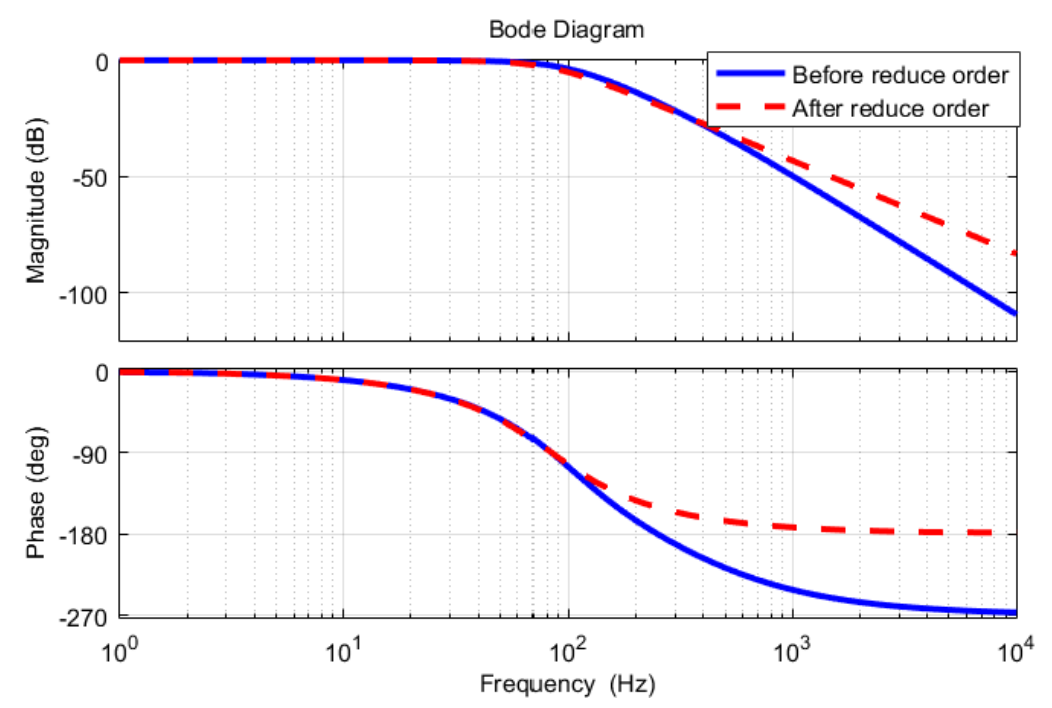

Fig. 1 Bode response

The Bode response before and after the order' change is shown is Fig.1. The curves coincide with each other at the middle and low frequency section before and after neglecting the highest order. It indicates that the lowered order model works well when the actual model works at middle and low frequency section.

After normalization of the highest order, the transfer function is given as follow:

$$
L(s)=\frac{k_{c} k_{v}}{s^{2}+b_{1} s}
$$

Here:

$$
k_{v}=k_{g}\left(T_{f}+p_{4}\right), \quad b_{1}=1 /\left(T_{f}+p_{4}\right)
$$

For the unit feedback position system, the closed loop transfer function is given as follow:

$$
\Phi(s)=\frac{k_{c} k_{v}}{s^{2}+b_{1} s+k_{c} k_{v}}
$$

$\Phi(s)$ is the ideal reference model of the FSM.

\section{Adaptive control law based on fast differential}

It is difficult to obtain the whole state variables of the FSM. But the adaptive control algorithm can also be formed by using the platform's input and output signal ${ }^{[8-9]}$. Supposing the generalized error is defined as the D-value between the reference model's output and the actual model's output, there is:

$$
e=\theta_{m}-\theta_{p}
$$

As is shown in Fig.2, the adjustable parameters of the controller can be set up by using the generalized error.

Supposing $\theta_{m}, \theta_{p}, \theta_{r}$ are the output position signal of the reference model, output position signal of actual model and the given input position signal, the differential equation of the inertial stabilized platform's reference model at the middle and low frequency section can be given as follow:

$$
\ddot{\theta_{m}}+a_{m 1} \dot{\theta}_{m}+a_{m 0} \theta_{m}=k_{0} \theta_{r}
$$




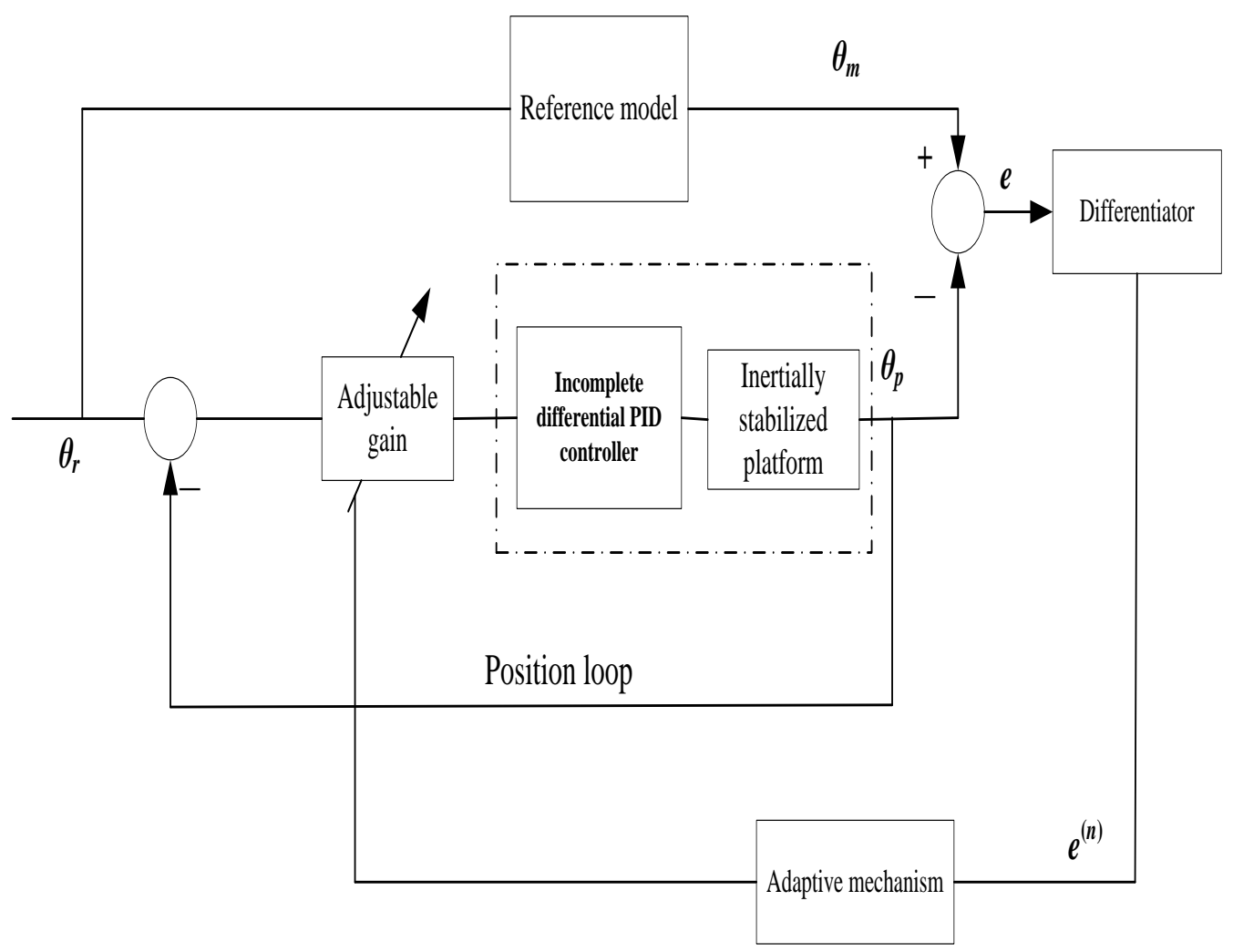

Fig. 2 Diagram of the control system

Here, $a_{m 1}, a_{m 0}, k_{0}$ are constant. The differential equation of the actual model at middle and low frequency section can also be given as follow:

$$
\ddot{\theta}_{p}+a_{p 1} \dot{\theta}_{p}+a_{p 0} \theta_{p}=k_{c} k_{v} \theta_{r}
$$

Here:

$$
a_{p 1}=b_{1}, \quad a_{p 0}=k_{c} k_{v}
$$

Supposing:

$$
e=\left[e_{1}, e_{2}\right]^{T}, \quad e_{1}=e, e_{2}=e_{1}
$$

The generalized error equation of the inertial stabilized is:

$$
\ddot{e}+a_{m 1} \dot{e}+a_{m 0} e=\delta_{1} \dot{\theta_{p}}+\delta_{0} \theta_{p}+\sigma \theta_{r}
$$

Here:

$$
\sigma=\left(k_{0}-k_{c} k_{v}\right), \quad \delta_{i}=a_{p i}-a_{m i}(i=0,1)
$$

Forming the equation (13) into vector differential equation form, there is:

$$
\dot{\mathbf{e}}=\mathbf{A e}+\Delta a+\Delta b
$$

Here:

$$
\mathbf{A}=\left[\begin{array}{cc}
0 & 1 \\
-a_{m 0} & -a_{m 1}
\end{array}\right] \quad \Delta a=\left(\begin{array}{l}
0 \\
\sum_{i=0}^{1} \delta_{i} \theta_{p}^{(i)}
\end{array}\right) \quad \Delta b=\left(\begin{array}{l}
0 \\
\sigma \theta_{r}
\end{array}\right)
$$

Supposing the parameter's error vector is:

$$
\Gamma^{T}=\left[\delta_{1}, \delta_{0}, \sigma\right]^{T}
$$


Lyapunov function is defined as follow:

$$
V=\frac{1}{2}\left[\mathbf{e}^{T} \mathbf{P e}+\Gamma^{T} \boldsymbol{\Lambda} \Gamma\right]
$$

Where, $\mathbf{P}$ is the symmetrical positive determined matrix, $\Lambda$ is the diagonal positive definite matrix. Supposing $\lambda_{0}, \lambda_{1}, \mu$ are the arbitrary positive number. Taking the derivative of $\mathrm{V}$, there is

$$
\dot{V}=\frac{1}{2} e^{T}\left(\mathbf{P A}+\mathbf{A}^{T} \mathbf{P}\right) e+\sum_{i=0}^{1} \delta_{i}\left[\lambda_{i} \dot{\delta}_{i}+\left(\sum_{k=1}^{2} e_{k} p_{k 2}\right) \theta_{p}^{(i)}\right]+\sigma\left[\mu \dot{\sigma}+\left(\sum_{k=1}^{2} e_{k} p_{k 2}\right) \theta_{r}\right]
$$

To insure $\dot{V}$ is negative definite, the symmetric positive definite matrix $\mathbf{Q}$ is chosen to satisfy the following equation:

$$
\mathbf{P A}+\mathbf{A}^{T} \mathbf{P}=-\mathbf{Q}
$$

At the same time, the second and third terms on the right side of the equation [18] should be equal to zero. Combining the upper equations, the adaptive control law can be got:

$$
\begin{gathered}
\dot{\sigma}=-\left(e_{1} p_{12}+e_{2} p_{22}\right) \theta_{r} / \mu \\
\dot{k}_{c}=\left(e_{1} p_{12}+e_{2} p_{22}\right) \theta_{r} /\left(\mu k_{v}\right)
\end{gathered}
$$

$p_{12}$ and $p_{22}$ are the elements of $\mathbf{P}$. Although there is an unknown gain $k_{v}$ in the formula, it can be treated as a constant because it multiplies with a free designed constant $\mu$. The formula contains the product of generalized error and input, and the generalized error and its differential term are also used.

In engineering, PSD can be directly used to measure the position signal of the stable platform, and then the differential signal of the generalized error signal can be obtained by using differential link. .However, because the essence of the differential link is a high pass filter, the performance of the control system will be seriously affected by the high frequency noise disturbance after adding the differential link. Even more, the introduction of the differential link may cause the instability of the control system. Therefore, we should avoid using differential links when obtaining differential signals in engineering.

Introduction of speed sensor is also feasible to get the platform's speed signal. The differential signal of the generalized error can also be obtained by subtracting the reference speed signal and the actual speed signal. But this method will not only increase the hardware cost and complexity of the control system but also the increasing noise input.

By using the differentiator, the denoised signal can be obtained and guided from the noise signal. Through the introduction of the fast differentiator into the MARC system, the generalized error signal and its differential value can be obtained, and then the control system without speed measurement can be designed.

A whole process fast differentiator is put forward in [10]. The expression of the differentiator is given as follow:

$$
\begin{aligned}
& x_{1}=x_{2} \\
& \dot{x}_{2}=R^{2}\left(-a_{0}\left(x_{1}-v(t)\right)-a_{1}\left(x_{1}-v(t)\right)^{m / n}-b_{0} \frac{x_{2}}{R}-b_{1}\left(\frac{x_{2}}{R}\right)^{m / n}\right)
\end{aligned}
$$

Here, $R>0, a_{0}, a_{1}, b_{0}, b_{1} \geq 0, m, n$ are the odd numbers larger than zero, and $\mathrm{m}<\mathrm{n} . x_{1}$ tracks the input signal $v(t) . x_{2}$ tracks the first-order derivative of, i.e. $v(t)$.

When $a_{1}=b_{1}=0$, linear differentiator plays the leading role. Put the generalized error signal as the input signal, the following equation can be get: 


$$
\begin{aligned}
x_{1} & =x_{2} \\
x_{2} & =R^{2}\left(-a_{0}\left(x_{1}-e(t)\right)-b_{0} \frac{x_{2}}{R}\right)
\end{aligned}
$$

After differential processing, the error signal and its first-order differential signal are given as follows:

$$
\begin{aligned}
& e_{1}=e(t)=x_{1}(t) \\
& e_{2}=e(t)=x_{2}(t)
\end{aligned}
$$

Taking the equation (24) into the equation (21), the design of error fast differential adaptive control law can be actualized.

\section{Simulation and analysis}

In order to explore the effectiveness of the presented control algorithm, numerical simulations are conducted in this section. The open loop response curve and its fitting curve of an experimental FSM which is obtained by scanning frequency method are shown in Fig.3. The transfer function of the FSM can be obtained by system identification. The open-loop transfer function of researched FSM is presented as follow:

$$
G(s)=\frac{0.6}{\left(1.055 e-5 s^{2}+6.507 e-4 s+1\right)(5.30 e-4 s+1)}
$$
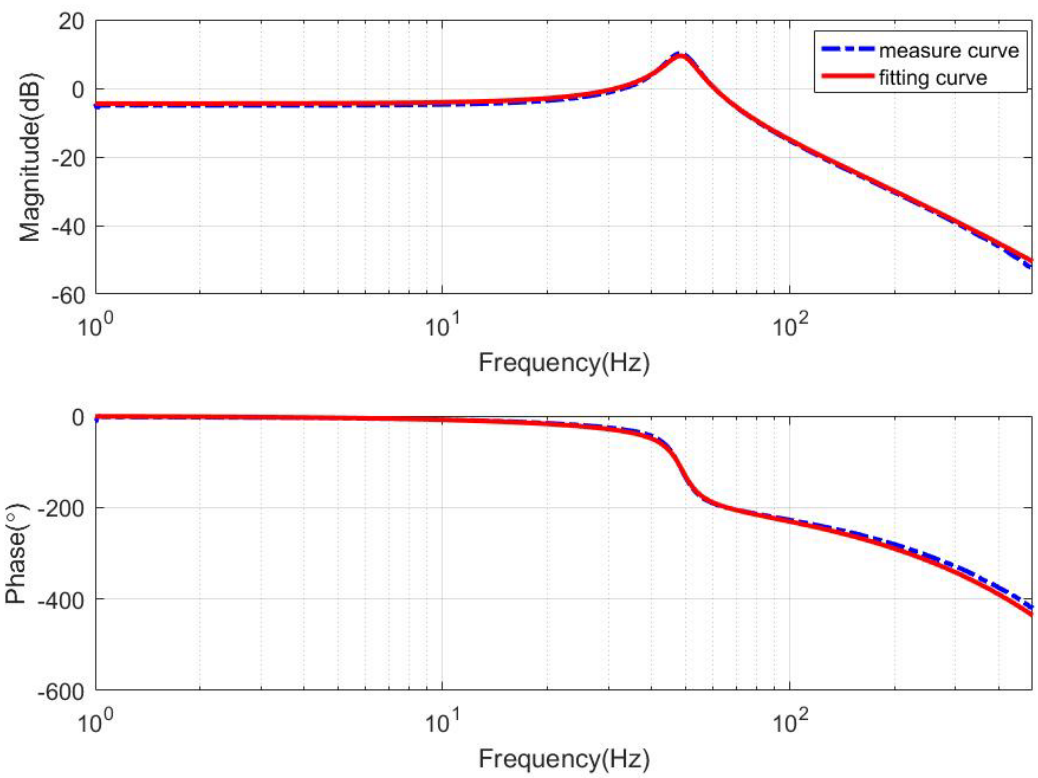

Fig.3 The position open-loop bode response

The $p_{4}$ in equation (1) reflects the change of the structural stiffness. $p_{4}$ will reduce if the structural stiffness weakens.

The parameters of the incomplete differential PID controller are given as follows:

$$
k_{p}=0.0551, k_{i}=591, k_{d}=0.0064, T_{f}=0.00078
$$

The parameters of the reference model and the actual model in model reference model control system are given as follows:

$$
\left\{\begin{array}{l}
a_{m 1}=769, \quad a_{m 0}=2.7 e 5, \quad k_{0}=2.7 e 5 \\
b_{1}=780, \quad a_{p 0}=3.0 e 5 \\
p_{12}=0.019, \quad p_{22}=6.502
\end{array}\right.
$$


The MARC system based on fast tracking differential is established in SIMULINK. And two sets of experiments are conducted to compare the incomplete differential PID control algorithm with the presented control algorithm:

(1) The tracking performance experiments of control system without considering measurement noise;

(2) The tracking performance experiments of control system when the sensor measured noise is taken into account.

The step response of the reference model, the incomplete derivative PID control algorithm and the MARC based on error fast differential are shown in Fig.4. It can be seen that the output of the incomplete differential control system is different from the reference model after the structural stiffness changes. And the step output curve of the adaptive control system error fast differential is basically coincided with the desired tracking curve after the changing. It indicates that the proposed algorithm can effectively track the desired system after stiffness changes without considering the measured noise.

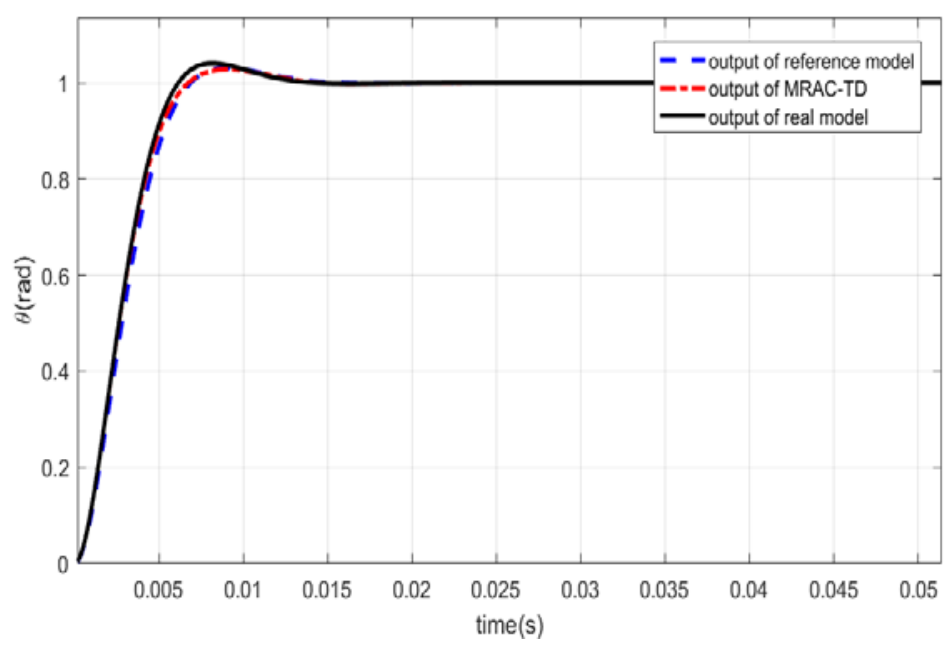

Fig.4 Step response without noise

The step response curves are shown in Fig.5. It can be seen that the output of the incomplete differential control system oscillates after the noise signal is applied, while the adaptive control system based on generalized error fast differential remains stable and could still track the expected curve.

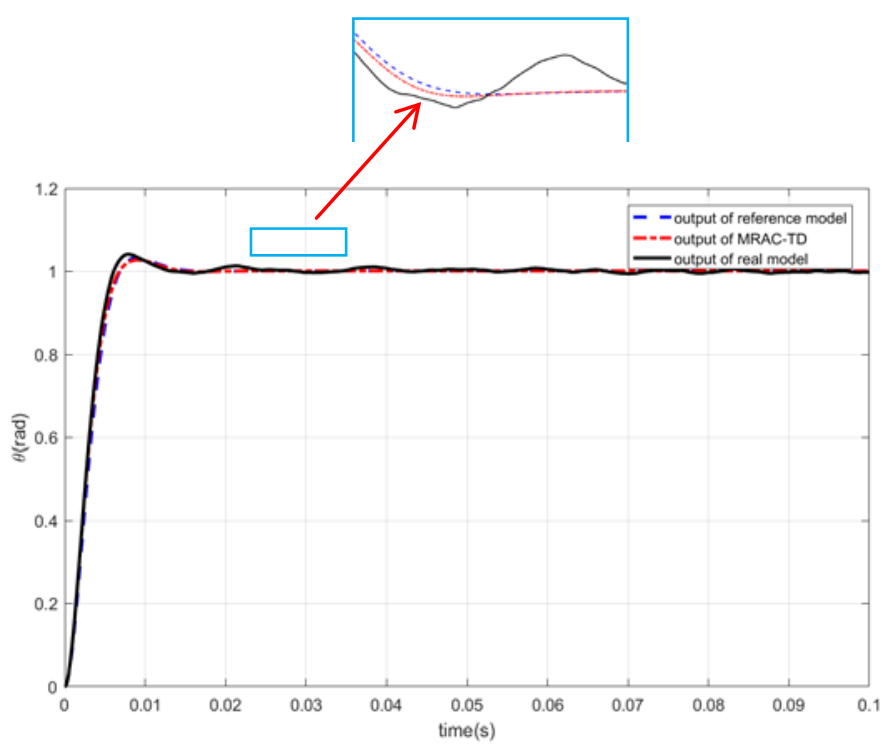

Fig.5 Step Response with measure noise

As is shown in the Fig.6, the angle deviation of the platform is compared with the two control 
methods. It can be seen from the diagram that the angle deviation signal of the incomplete differential control system is noisy under the sensor measurement noise, while the adaptive control based on error fast differential can completely suppress the influence of the sensor measurement noise. The simulation results show that the proposed control method can achieve high precision position tracking control with the flexible structure's change. The method can also effectively restrain the influence of sensor noise.

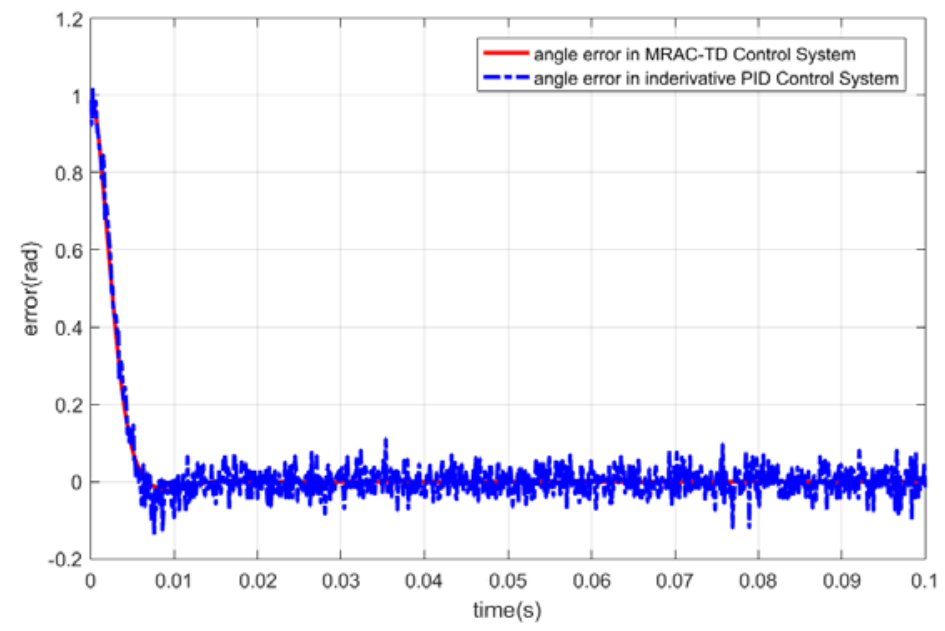

Fig.6 Angle error

\section{Conclusion}

The stiffness of the flexible support in FSM with VCM driver can easily change in working process, and the use of sensor will introduce the measurement noise when measuring the position. All of these questions make the output's uncertainty of the FSM increasing. Aimed at these problems, MRAC system based on fast differential is proposed in this paper by combining the adaptive control theory with tracking differential theory. Firstly, the mathematical model of the FSM control system is optimized to match the mathematical model of the tracking differentiator. Secondly, according to the input and output data of the FSM system, the adaptive control system is designed based on Lyapunov stabilized theory. Finally, the denoised system's generalized error signal and its differential signal are obtained by using a fast differentiator. The simulation results shows the FSM control system can achieve high precision tracking control after the flexible structure changes and the existence of measurement noise.

\section{References}

[1] Hilkert J M, Fast steering mirror technology: concepts and principle, IEEE Control Systems Magazine. 2008(12) 26-46.

[2] Masten M K, Fast steering mirror for optical imaging system: tracking dynamic targets with mobile sensors. IEEE Systems Magazine. 2008(12) 47-64.

[3] Cong S, Sun G L, Deng K, et al, Active disturbance rejection and filter control of gyro-stabilized platform, Optics and Precision Engineering. 2016, 24(1) 169-176

[4] Tian J, Deng C, Cao Z, et al, Application of Acceleration Feedback Techniques on Inertial Stabilization Platform, Opto-Electronic Engineering. 2016, 43(12) 40-45

[5] LI X T, Zhang B, Shen H H, Improvement of isolation degree of aerial photoelectrical stabilized platform based on ADRC, Optics and Precision Engineering. 2014, 22(8) 2223-2231.

[6] Li M, Li J, Modeling and simulation of unbalance disturbance for fast steering mirror, Journal of Chinese Inertial Technology. 2014, 22(4) 463-468. 
[7] Ai Z W, Tan Y, Wu Q Y, et al, Design of control parameters for fast steering mirror by improving root locu,. Laser Technology. 2017, 42(4) 558-561.

[8] Lv W S, Wang F, Li Y, Adaptive finite-time tracking control for nonlinear systems with unmodeled dynamics using neural networks, Advances in Difference Equations (2018).

[9] Chen X Y, Cao J D, Qiu J L, et al, Adaptive control of multiple chaotic systems with unknown parameters in two different synchronization modes, Advances in Difference Equations. 2016(1) $1-17$

[10] Wang X H, Chen Z Q, Yuan Z Z, Nonlinear tracking_differentiator with high speed in whole course, Control Theory \& Applications. 2003, 20(6) 87 